\section{IN THE NEWS}

A strange taste in music

Synaesthesia, in which one or more of our senses is stimulated by another, has long been recognized; now, for the first time, it has been shown to assist in the performance of a cognitive task. Lutz Jäncke and his colleagues at the University of Zürich have been studying a musician who not only sees colours when she hears a particular note - a fairly common form of synaesthesia - but also tastes them.

For Elizabeth Sulston, different tone intervals have different tastes, including salty, creamy and that of mown grass. She says of her synaesthesia, "I ... really became conscious of it at sixteen. Then I started to use it for the tone-interval identification" (news@nature.com, 2 March 2005). And, according to Michaela Esslen, one of Jäncke's co-workers, "She doesn't imagine the taste, she really tastes it" (ABC.net, 3 March 2005).

Jäncke and his colleagues compared her ability to correctly identify a tone interval with concurrent delivery to her tongue of either the taste she normally associates with that interval or a conflicting taste. Although incongruent tastes did not result in incorrect answers, they did affect the speed at which she was able to answer. Furthermore, the correct taste allowed her to recognize an interval more quickly than five non-synaesthetic musicians.

How this discovery might relate to the wider field of neuroscience is not certain. Jäncke points out that associations can aid memory, and adds, "It may also demonstrate that synaesthesia may be modified for learning and used for other things" (news@nature.com).

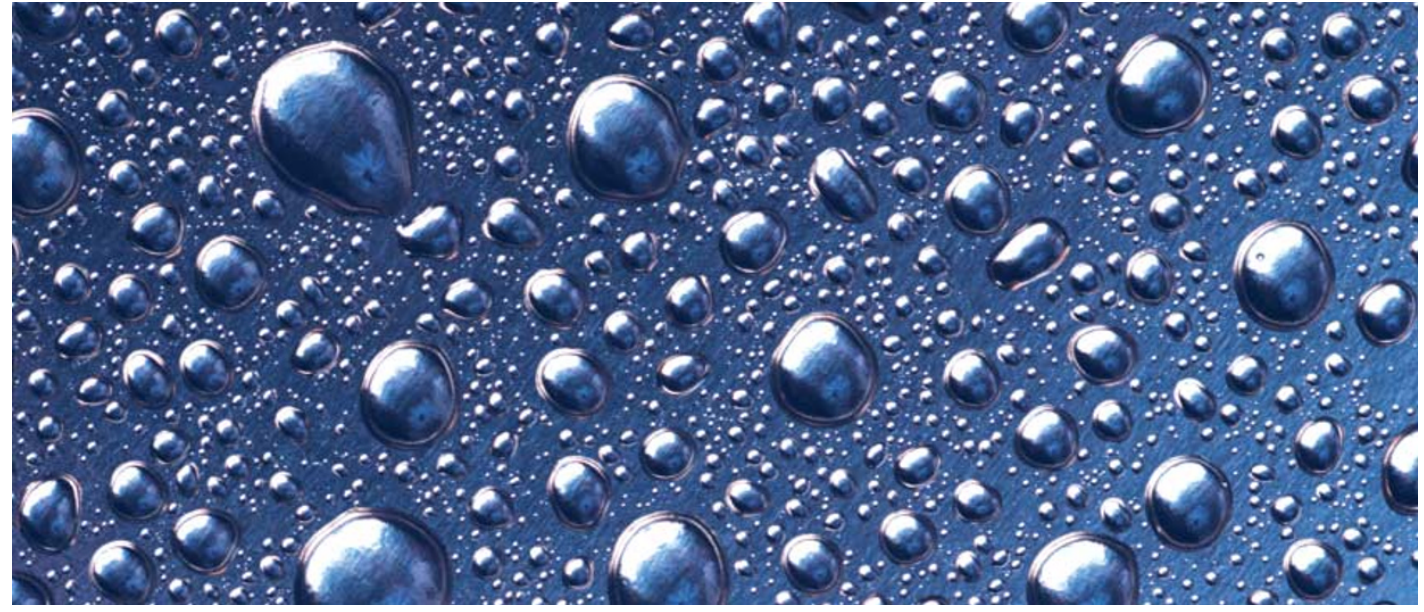

SYNAPTIC PHYSIOLOGY

\title{
Pools of transmission
}

Neurons can release synaptic vesicles and discharge

their neurotransmitter content either spontaneously, when they are at rest, or after they are stimulated and are actively relaying a message. In both cases it was thought that the same synaptic vesicles were responsible for releasing neurotransmitter. However, the authors of a new study published in Neuron suggest that two functionally segregated pools of synaptic vesicles might be involved in neurotransmission under different circumstances.

Activity-dependent neurotransmitter release is a highly regulated and precisely timed process, whereas spontaneous fusion of synaptic vesicles with the presynaptic membrane depends only loosely on factors such as calcium concentration and neuromodulators.

This dichotomy has led to a debate about the mechanism and location of spontaneous vesicle fusion. To address this issue, Sara and colleagues visualized vesicle trafficking by incubating mature hippocampal cultures with a styryl dye that indiscriminately labelled endocytosing membranes and an antibody, Syt1, that was generated against the luminal domain of the synaptic vesicle protein synaptotagmin 1 .

The Syt1-labelled spontaneous vesicles were then stained with a fluorescently conjugated secondary antibody, which, in non-permeabilized cells, could recognize the primary antibody only once it had been exposed to the extracellular space. Syt1 was detected both intracellularly and on the plasma membrane, regardless of whether live or fixed and permeabilized cells were used. Under resting conditions, spontaneously labelled vesicles showed more pronounced destaining (the dye was lost on exocytosis) than their activity-dependent counterparts. These findings indicate that spontaneous vesicles recycle at rest and are more likely to be reused spontaneously.

To investigate whether spontaneous vesicles are recruited for activity-dependent neurotransmission, the authors measured destaining of spontaneously loaded vesicles after depolarization or the application of action potentials at $10 \mathrm{~Hz}$. Stimulation resulted in much slower destaining of the spontaneously stained puncta compared with that of activity-dependent vesicles, which indicates that, after spontaneous exo-endocytosis, synaptic vesicles constitute a reserve pool that is only reluctantly available for release. This was confirmed by the fact that blocking vesicle refilling at rest led to a sixfold reduction in the frequency of spontaneous miniature synaptic currents without significantly affecting the size of evoked synaptic currents.

What is the molecular mechanism that separates these two pools of synaptic vesicles? As yet there is no definitive answer, but some clues have come from studies of synaptobrevin 2-deficient mice.

Synaptobrevin 2 is the main synaptic vesicle SNARE protein and has an important role in membrane fusion. In synaptobrevin 2-deficient hippocampal cultures, there were no significant differences in destaining between spontaneous and activitydependent vesicles - either at rest or after stimulation - which indicates that synaptobrevin 2 might be involved in the segregation of the two vesicle pools.

These findings lead us to question one of the core tenets of synaptic function and reveal interesting intricacies in the organization of synaptic vesicles within individual synapses. It will be important to determine whether the two sets of synaptic vesicles have different molecular compositions and whether their recycling pathways are differentially regulated.

Jane Qiu

(1) References and links

ORIGINAL RESEARCH PAPER Sara, Y. et al. An isolated pool of vesicles recycles at rest and drives spontaneous neurotransmission. Neuron $\mathbf{4 5}$, 563-573 (2005)

FURTHER READING Rizzoli, S. O. \& Betz, W. J. Synaptic vesicle pools.

Nature Rev. Neurosci. 6, 57-69 (2005)

WEB SITE

Kavalali's laboratory:

http://www.utsouthwestern.edu/findfac/professional/0,2356,42113,00.html 